The whole question is of great interest, and I shall pursue the inquiry further. Meanwhile, I have written this note in the hope that others, better acquainted with the magnetic and geophysical aspects of the problem, may pass their judgment.

EDWARD S. KING.

Harvard College Observatory,

Cambridge, Massachusetts,

Nov. 21.

\section{Oxide Films responsible for the Tints on Heated Copper.}

Much has been published on the tints of heated copper, but disagreement prevails regarding the oxide responsible for the colours; Dunn (Proc. Roy. Soc., $111[\mathrm{~A}], 211$; 1926) apparently attributes them to cuprous oxide, and Constable (Ibid., 115 [A], 583 ; 1927) to a "veneer of cupric oxide." Recently the oxide films have been isolated from their basis, the metal being dissolved from below by anodic treatment in concentrated potassium sulphate solution; the oxide films, thus undermined, peel off in curling flakes, which retain the grooves and ridges left by the abrasive treatment used to clean the copper before oxidation. The thicker films can also be removed mechanically.

The two oxides present in the films are quite different in appearance and can be distinguished by chemical tests. At thicknesses above the interference colour range, cuprous oxide films appear brown by transmitted light and exhibit by reflected light a characteristic colour best described as pale chocolate; but a veneer of cupric oxide produces a dark grey reflex without a trace of brown or red. Residual metallic copper, where it exists in the films, has a bright red lustre and is opaque ; its presence may be revealed by the action of silver nitrate, which produces a microscopic 'silver tree.'

It has been found that the colours are due to cuprous oxide. Cupric oxide is indeed formed under strongly oxidising conditions, but it obscures the colours, and must be avoided if the later tints are to be obtained. Thus Constable, working under conditions favourable to the production of cupric oxide, obtained no colours beyond the middle of the second order, the tints darkening and passing into the black characteristic of cupric oxide. This has been confirmed, but it was found that if the formation of cupric oxide is avoided, the sequence can be followed to the fourth order; the tints then pass gradually into the characteristic colour of cuprous oxide. The easiest way to prevent the formation of cupric oxide is to use a mildly oxidising gas mixture-preferably obtained from a flame of pure alcohol.

Within the interference-colour range, the cuprous oxide films are quite transparent. On the whole, the oxide film taken from copper tinted to an early colour is more transparent than that taken from copper displaying a high-order tint; but in the latter case fragments of thin, highly transparent films are also separated along with the thicker skin. This is apparently due to the fact that the skin cracks as it thickens, allowing air access to the metal exposed at the crack, so that another film is formed below the first; this lower film will be generally thinner than the first and will diminish in thickness with the distance from the crack. The formation (at a crack) of one skin below another has been directly observed at high temperatures, and there is evidence that the phenomenon is general ; for it is found that copper heated rather too strongly for interference colours nevertheless yields-on stripping-flakes which display bright colours, the tints varying from place to place as the result of varying thickness.

No. 3088, Vor. 123]
The colours of the stripped films are often brilliant by reflected light, rose, blue, and green hues being obtained; by transmitted light the interference tints are largely masked by the yellow hue due to selective absorption, but there is a slight variation of colour with thickness between yellowish-green, bright yellow, and brown.

The films isolated from copper tinted to the early first-order colours usually contain opaque spots due to included metallic copper, and the metallic residue increases on passing to films taken from copper heated insufficiently to produce colours. In films removed from copper merely exposed (after abrasion) to dry air at ordinary temperatures, the opaque areas generally predominate over the transparent areas, although the character of the composite oxide-metal layer varies with the nature of the abrasive treatment employed. The composite layer appears to be formed as follows: Abrasion produces a network of cracks, increasing the true surface area, as found by Bowden (NATURE, 112, 647; 1928). On exposure to air, the walls of these cracks become oxidised, and the internal oxide-sheaths obstruct to a large extent the anodic removal of metallic copper, so that the layer left (after the unchanged basis has been dissolved away) consists of both metal and oxide. Clearly with increasing temperature or time of exposure to oxygen, the proportion of residual metal in the layer stripped will diminish, and hence the films obtained from copper tinted to any of the later colours are practically free from metal.

$$
\text { Ulick R. Evans. }
$$

University Chemical Laboratory, Cambridge.

\section{Radio Echoes and Conditions for their Occurrence.}

Since October 24, the emission of signals (see Nature, Nov. 3 and Dec. 8, 1928) from the shortwave emitter PCJJ (Holland) has been continued twice a week, and sometimes more frequently. Through the Norwegian Telegraph Office a series of receiving stations has maintained a continual watch, and an oscillograph has been ready for use at all times, but no echoes have been heard, either in Norway or in Eindhoven.

It appears from this, and from the long silence during experiments in the spring and summer, that the echoes constitute a very rare phenomenon and owe their occurrence to a series of favourable coincident circumstances. The wave-length must be the most favourable one, and the emission must be sent out in the right direction and with sufficiently great energy. (A transmitter station in the tropics would probably be better than a station in Holland.) The Kennelly-Heaviside layer must be penetrable by the outgoing and returning waves, and must also be favourable for the hearing of both signals and echoes, and the receiving apparatus must be sufficiently sensitive and exactly adjusted.

Further, there must be good conditions for hearing without too many atmospheric disturbances, and, last but not least, the emission of electrons from the sun must take place in such a way that reflecting surfaces in space outside the orbit of the moon may be formed and may have the most favourable shape for a good reflection of the waves.

As regards the last point, the mathematical theory of the motion of electric corpuscles around a magnetised sphere shows that the chances of obtaining a well-defined toroidal space round the earth are good when the direction to the sun lies near the magnetic equatorial plane (perpendicular to the magnetic axis). 